\title{
Responsive Copolymers for Enhanced Petroleum Recovery
}

$\mathrm{DOE} / \mathrm{BC} / 14882--2$

\author{
Quarterly Technical \\ Progress Report
}

DE93 014531

\section{December 22, 1992 - March 21, 1993}

\section{DE-AC22-92BC 14882}

by

Roger Hester and Charles McCormick

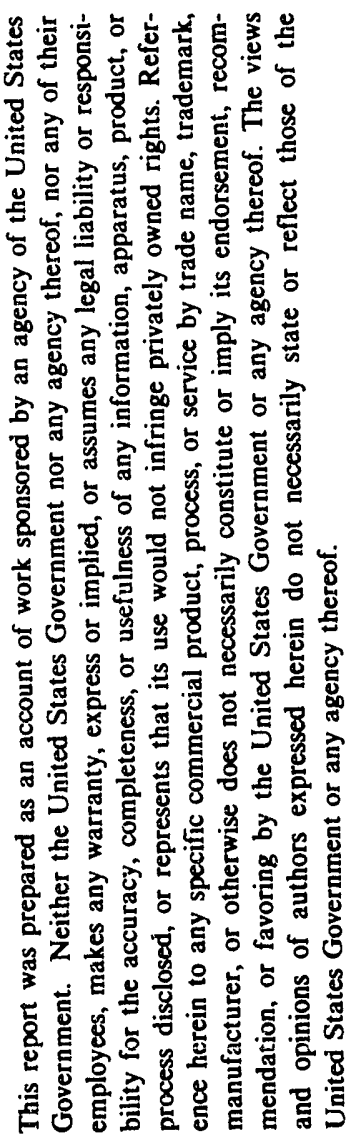

$$
\begin{gathered}
\text { The University of Southern Mississippi } \\
\text { Hattiesburg, MS 39406-0076 }
\end{gathered}
$$

Departme'nt of Polymer Science

Contract Begin Date-September 22, 1992

Contract First Year End Date-September 21, 1993

Current Year DOE Award- $\$ 273,400$
RECEIVEDD

JUN 071993

OSTI

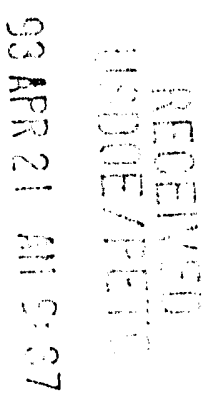

Contracting Officer's Representative-Jerry $\cdot$ F. Casteel 


\section{Project Objective}

The overall goal of this research is the development of advanced water-soluble copolymers for use in enhanced oil recovery which rely on reversible microheterogeneous associations for mobility control and reservoir conformance.

\section{Summary of Technical Progress}

\section{A. Task 1-Advanced Copolymer Synthesis}

During the quarter, synthetic efforts have centered on preparation of copolymers of acrylamide with a hydrophobic cationic comonomer, DAMAB. These copolymers bind anionic surfactants strongly to form viscous solutions.

\section{Monomer Synthesis}

Scheme 1 illustrates the synthesis of hydrophobically-modified acrylamido monomer N,N-dimethyl-N-dodecyl-N-(2-acrylamidoethyl) ammonium bromide. Into a 250 $\mathrm{ml} 3$-neck round-bottom flask were placed methylene chloride $(82 \mathrm{ml}), \mathrm{N}, \mathrm{N}$ dimethylethylenediamine $(8.82 \mathrm{~g}, 0.100 \mathrm{~mole})$, and $6 \mathrm{~N}$ sodium hydroxide $(25 \mathrm{ml})$. The mixture was placed in an ice bath and agitated by a magnetic stirrer under nitrogen atmosphere. When the temperature dropped below $5^{\circ} \mathrm{C}$, acryloyl chloride $(9.36 \mathrm{~g}, 0.104$ mole) in $20 \mathrm{ml}$ of methylene chloride was added slowly from an addition funnel to maintain the temperature below $10^{\circ} \mathrm{C}$. The mixture was stirred for an additional 30 minutes after complete addition of acryloyl chloride. The organic layer was then separated, washed twice with water and once with concentrated $\mathrm{NaCl}$ solution, and dried over anhydrous sodium sulfate. The solvent was removed on a rotary evaporator to yield a slightly yellow oil ( $11.67 \mathrm{~g}, 80 \%$ yield). The crude product was purified by vacuum distillation in the presence of a small amount of phenothiazine as an inhibitor. A colorless oil was collected at $88--90^{\circ} \mathrm{C}$ under vacuum of $0.5 \mathrm{~mm}-\mathrm{Hg}$. IR $(\mathrm{KBr}) 3284(\mathrm{~N}-\mathrm{H})$, $1655(\mathrm{C}=\mathrm{O}) \mathrm{cm}^{-1} ;{ }^{1} \mathrm{H} \mathrm{NMR}\left(\mathrm{CDCl}_{3}\right) \delta 2.23(\mathrm{~s}, 6 \mathrm{H}), 2.46(\mathrm{t}, 2 \mathrm{H}), 3.42(\mathrm{~m}, 2 \mathrm{H}), 5.55-5.60$ $(\mathrm{m}, 2 \mathrm{H}), 6.25-6.27(\mathrm{~m}, 4 \mathrm{H}), 7.53(\mathrm{br}, 1 \mathrm{H}) ;{ }^{13} \mathrm{C} \mathrm{NMR}\left(\mathrm{CDCl}_{3}\right) \delta 36.45,44.43,57.29$, $124.61,130.59,165.05$.

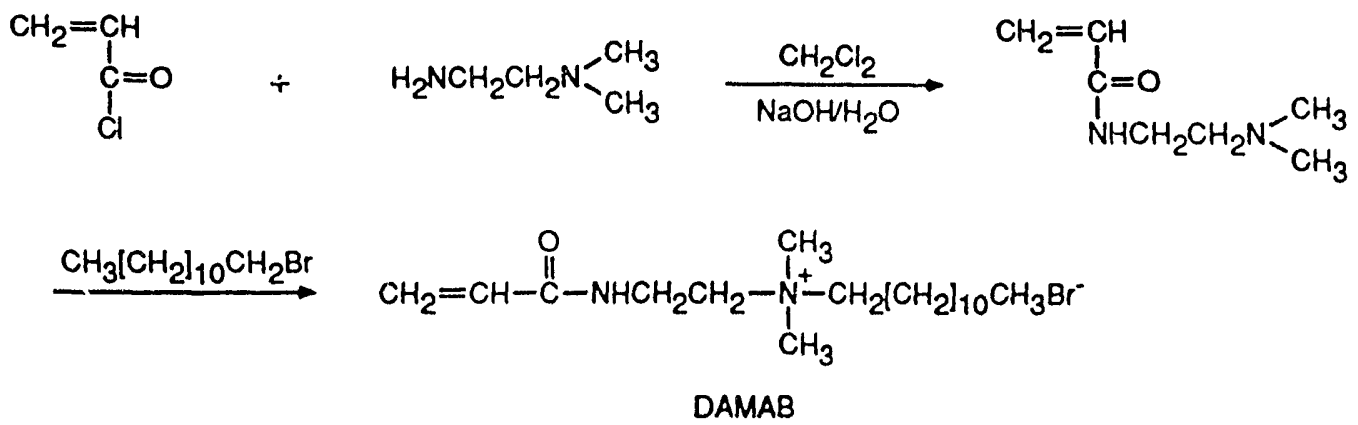

Scheme 1. Synthesis of DAMAB Monomer. 
Freshly distilled N-(N,N-dimethylaminoethyl)acrylamide ( $5.0 \mathrm{~g}, 0.035$ mole) was then treated with dodecylbromide ( $13 \mathrm{~g}, 0.052$ mole) under nitrogen at room temperature for 48 hours. Exces dodecylbromide was decanted and the transparent gel remaining was placed in a freezer overnight. The resulting white solid was washed with two portions of ether, and dried under vacuum. Further purification of the final product was accomplished by recrystallization from a $1: 1$ mixture of acetone and ether. Yield: 13.26 g (96\%). m.p. 70.5--72 ${ }^{\circ} \mathrm{C}{ }^{1} \mathrm{H} \mathrm{NMR}\left(\mathrm{CDCl}_{3}\right) \delta 0.88(\mathrm{t}, 3 \mathrm{H}), 1.25-1.34(\mathrm{br} \mathrm{m}, 20 \mathrm{H}), 1.76$ (m, $2 \mathrm{H}), 3.42(\mathrm{~s}, 6 \mathrm{H}), 3.82(\mathrm{~m}, 4 \mathrm{H}), 5.63-5.74(\mathrm{~m}, 1 \mathrm{H}), 6.28-6.48(\mathrm{~m}, 2 \mathrm{H}), 8.79(\mathrm{br}, 1 \mathrm{H}) ;{ }^{13} \mathrm{C}$ $\operatorname{NMR}\left(\mathrm{CDCl}_{3}\right) \delta 13.18,21.71,21.97,25.36,28.36,28.55,28.65,30.94,33.18,50.89,51.18$, $64.39,165.56$.

\section{Copolymerization in the Absence of External Surfactant}

To a $1000 \mathrm{ml}$ 3-necked round bottom flask equipped with a mechanical stirrer, a condenser, and a nitrogen inlet were added acrylamide (AM) and N,N-dimethyl-N-dodecyl$\mathrm{N}$-(2-acrylamidoethyl) ammonium bromide (DAMAB) in the desired ratio, and $500 \mathrm{ml}$ deionized water. The total concentration of the monomers was kept $0.21 \mathrm{M}$. The solution was heated to $50^{\circ} \mathrm{C}$ in a water bath with a small nitrogen stream passing through the system. Polymerization was then initiated by addition of $\mathrm{K}_{2} \mathrm{~S}_{2} \mathrm{O}_{8}\left(0.0262 \mathrm{~g}, 9.71 \times 10^{-5}\right.$ mole in $3 \mathrm{ml}$ of deionized water) via a syringe. Polymerization was conducted continuously at $50^{\circ} \mathrm{C}$ for 6 hours and then the reaction stopped by precipitating the polymer in $800 \mathrm{ml}$ of acetone. The precipitated polymer was washed twice with acetone and vacuum dried. Conversion was 60--72\%. Further purification was accomplished by redissolving the polymer in water and dialyzing against water using a 12,000--14,000 molecular weight cut-off dialysis tubing for a week. The polymer was recovered by freeze drying.

\section{Copolymerization in the Presence of Surfactant}

Equimolar amounts of cetyltrimethylammonium bromide and DAMAB monomer were added with acrylamide to the polymerization system. The same procedure as in the previous case was followed for polymerization and polymer purification.

\section{Solution Polymerization}

A copolymer containing 5 mole \% DAMAB in the feed was prepared via homogeneous solution polymerization. AM (23.3 g, 0.328 mole) and DAMAB $(6.79 \mathrm{~g}$, $0.0173 \mathrm{~mole}$ ) were dissolved in $300 \mathrm{ml} \mathrm{t}$-butanol. The solution was deoxygenated with nitrogen for 30 minutes at $50^{\circ} \mathrm{C}$. AIBN $\left(0.056 \mathrm{~g}, 3.4 \times 10^{-4}\right.$ mole $)$ was then added to initiate the polymerization. Polymerization was conducted for 10 hours. In this case, polymer precipitated from solution as polymerization continued. Purification procedure was as described for the heterogeneous polymerization. A quantitative yield was obtained.

\section{B. Task 2-Characterization of Molecular Structure}

${ }^{1} \mathrm{H}$ and ${ }^{13} \mathrm{C}$ NMR spectra were recorded using a Bruker AC-300. A Mattson 2020 Galaxy Series FTIR was used to obtain infrared spectra. Surface tension measurements 
were performen with a Kruss Processor tensiometer $\mathrm{K} 12$ instrument at $25^{\circ} \mathrm{C}$. Viscosity measurements were conducted with a Contraves LS-30 low shear rheometer at a constant shear rate of 6 reciprocal seconds at $25^{\circ} \mathrm{C}$. Classical light scattering studies were performed on a Chromatix KMX-6 low-angle laser light scattering spectrophotometer with a $2-\mathrm{mW} \mathrm{He}-\mathrm{Ne}$ laser operating at $633 \mathrm{~nm}$. Refractive index increments $(\mathrm{dn} / \mathrm{dc})$ were obtained using a Chromatix KMX-16 differential refractometer. Steady-state fluorescence measurements were made with a Spex Fluorolog-2 fluorescence spectrometer. Elemental analyses to determine hromine content were conducted by MHW Laboratories of Phoenix, Arizona. Characterization results are summarized in the table below.

Structural Parameters of the Copolymers

\begin{tabular}{||c|c|c|}
\hline \multicolumn{1}{|c|}{ Copolymer } & $\begin{array}{c}\text { DAMAB Content } \\
\text { (mole \%) }\end{array}$ & $\mathrm{M}_{\mathrm{w}} \times 10^{-6}$ \\
\hline \hline PAM & 0 & $1.12^{\mathrm{a}}$ \\
\hline R-C12-1 & 0.32 & $0.74^{\mathrm{a}}$ \\
\hline BS-C12-4.3 & 4.3 & $0.95^{\mathrm{b}}$ \\
\hline B-C12-4.7 & 4.7 & $1.04^{\mathrm{b}}$ \\
\hline R-C12-5.1 & 5.1 & $0.46^{\mathrm{b}}$ \\
\hline B-C12-10.5 & 10.5 & $1.12^{\mathrm{b}}$ \\
\hline
\end{tabular}

Measurement was taken in deionized water.

${ }^{b}$ Measurement was taken in $\mathrm{MeOH} / \mathrm{H}_{2} \mathrm{O}$ mixture (50/50 by volume).

\section{Task $3-$ Solution Rheology}

In the last progress report, relationships were developed to estimate a polymer solution's resistance to movement in a porous media due to extension of the polymer. ${ }^{1}$ Solution resistance increased with polymer molecular weight, higher fluid velocities and porous media geometries which favored formation of extensional flow fields. For porous media composed of uniformly packed spheres, the sphere diameter defined the geometry and the propensity to produce extensional flow conditions.

The effects of the above parameter on fluid flow resistance were expressed by

$$
\Psi=\Psi_{\max }^{e}
$$

where $\Psi$ is the normalized solution mobility, $\Psi_{\max }$ is the maximum mobility change per volume fraction of polymer coils, and $\varepsilon$ is an exponent which controls the extent to which the maximum mobility change conditions develop. $\Psi_{\max }$ was shown to be a function of polymer molecular properties and concentration. The exponent $\varepsilon$ is a function of a 
Deborah Number, $\mathrm{N}_{\mathrm{De}}$, which is a product of elongation rate, $\Gamma$, and polymer molecular response time, $\tau$. When $\mathrm{N}_{\mathrm{De}}$ increases, $\varepsilon$ approached unity and $\Psi$ approached $\Psi_{\max }$.

$$
\varepsilon=\frac{1+\operatorname{erf}\left(\frac{N_{D_{e}}-\mu}{\sigma}\right)}{2}
$$

Equation 2 defines $\varepsilon$. The dimensionless parameters $\mu$ and $\sigma$ adjust for local variation of the porous media structure and/or the disiribution of polymer molecular weights found in the solution.

We will now expand the above analysis to sandstone reservoirs. These reservoirs have porosities, $\phi$, which range from 0.1 to 0.4 and permeabilities, $k$, which vary from 1.0 $\times 10^{-10}$ to $3.0 \times 10^{-8} \mathrm{~cm}^{2}$ (10 to $3000 \mathrm{md}$ ). For many sandstone reservoirs, $\phi$ can be related to $\mathrm{k}$ in the following manner.

$$
\phi=a+b \log k
$$

The parameters $\mathrm{a}$ and $\mathrm{b}$ vary with the particular reservoir. Woodbrine sandstone values for $\mathrm{a}$ and $\mathrm{b}$ are 0.465 and 0.025 , respectively, where $\mathrm{k}$ is expressed in $\mathrm{cm}^{2}$ dimensions. ${ }^{2}$

Although a sandstone reservoir is composed of compressed or consolidated sand particles, this porous media can be crudely characterized by an average sand sphere diameter, $\mathrm{d}$. This diameter can be estimated from $\phi$ and $\mathrm{k}$ values of the reservoir using the Kozeny-Carman relationship. ${ }^{3}$

$$
\mathrm{d}=\frac{1-\phi}{\phi} \sqrt{\frac{180 \mathrm{k}}{\phi}}
$$

The reservoir characteristic particle diameter, $d$, can be used to estimate the extension rate, $\Gamma$, for a fluid forced through the media at velocity, $v$. As previously discussed $^{1}$,

$$
\Gamma=\frac{\sqrt{2}}{\phi} \frac{v}{d}
$$

The fluid velocity in the porous media, $v$, varies with distance from the wellhead. For cylindrical wells having volumetric injection rates $Q$ through a pay length of $L$, the local fluid velocity at radial distance $\mathbf{r}$ can be calculated.

$$
v=\frac{Q}{2 \pi r L}
$$

Combination of Equations 4, 5 and 6 shows that the fluid extension rates in sandstone near the wellhead can be determined by 


$$
\Gamma=\frac{Q}{\pi \mathrm{rL}(1-\phi)} \sqrt{\frac{\phi}{360 \mathrm{k}}}
$$

If one uses a relationship such as Equation 3 to define porosity in terms of permeability, then the fluid extension rate is a function of only well injection rates, $Q / L$, reservoir permeability, $k$, and radial distance from the well, $r$.

The above relationship was used with Equation 1 to model the relative near well reservoir resistance to injection of high molecular weight polyacrylamide solutions. Using this model, Figure 1 was constructed to show the solution to solvent resistance ratios, $f / f_{s}$, as a function of polymer molecular weight, $M$, solution dimensionless concentration, $[\eta] \mathrm{C}$, reservoir permeability, $k$, and radial distance from the wellhead, $r$. A constant injection rate of 20 barrels per day per foot of pay was used to construct this figure.
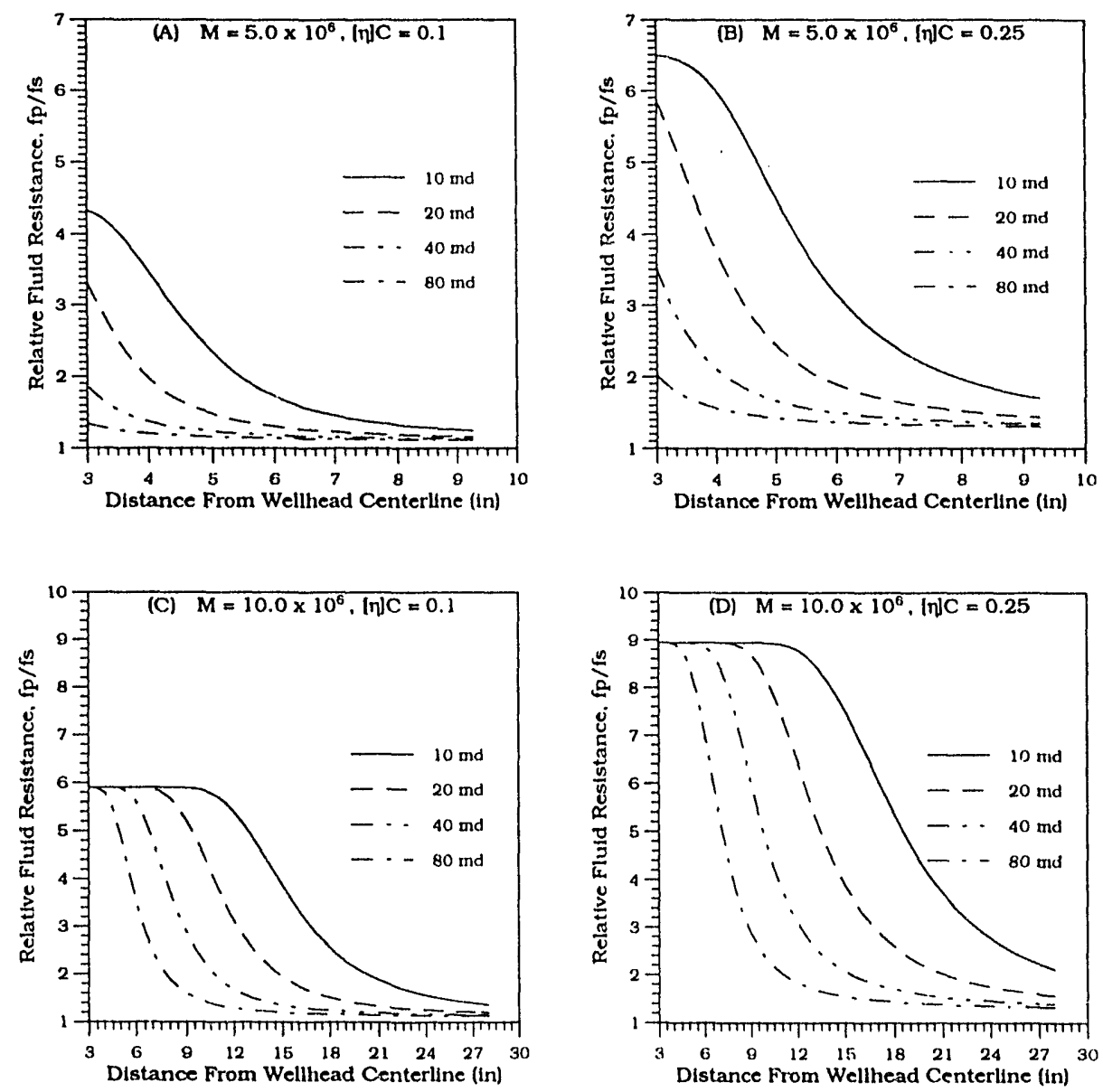

Figure 1. Predicted polyacrylamide dilute solution flow resistance in typical Woodbrine sandstones near a 6 inch diameter wellhead at injection rates of 20 barrels per day per foot of pay. 
Examination of Figure 1 reveals the following:

1. Extension of polymer molecules and thus increased fluid flow resistance only occurs very near the wellhead.

2. The total fluid flow resistance, which is proportional to the area under a curve increases with polymer molecular weight (compare condition $A$ to $C$ aind $B$ to $D$ ), polymer concentration (compare condition $A$ to $B$ and $C$ to $D$ ) and lower reservoir permeabilities.

3. Complete polymer extension and thus, maximum fluid resistance, can easily develop with typical injection condition. This is shown by the flattening of the curves to zero slope under conditions $C$ and $D$.

4. Although not shown by Figure 1, an increase in wellhead fluid injection magnifies fluid flow resistance significantly.

The above analysis was done for injection of dilute polymer solutions ([n]C $\leq 0.25$ ). Many of the arguments used to develop this extension model are less accurate or invalid as polymer concentration increases. Usually, polymer flooding solutions are much more concentrated $([\eta] C>0.50)$. Under more concentrated conditions, fluid resistance due to polymer coil extension is expected to be much greater. Injection conditions using concentrated solutions can degrade the polymer because extensional forces exceed macromolecular covalent bonding forces. Future efforts will expand and experimentally validate the dilute solution extension model for more concentrated solutions and also explore polymer degradation in extensional flow fields.

\section{$\underline{\text { References }}$}

1. C. L. McCormick and R. D. Hester, "Responsive Copolymers for Enhanced Petroleum Recovery," Progress Report, December 22, 1992.

2. R. E. Collins, Flow of Fluids Through Porous Materials, Petroleum Publishing Company: Tulsa, 1976; p. 17.

3. A. E. Scheidegger, The Physics of Flow Through Porous Media, 3rd Ed., University of Toronto Press: Toronto, 1974; p. 141. 

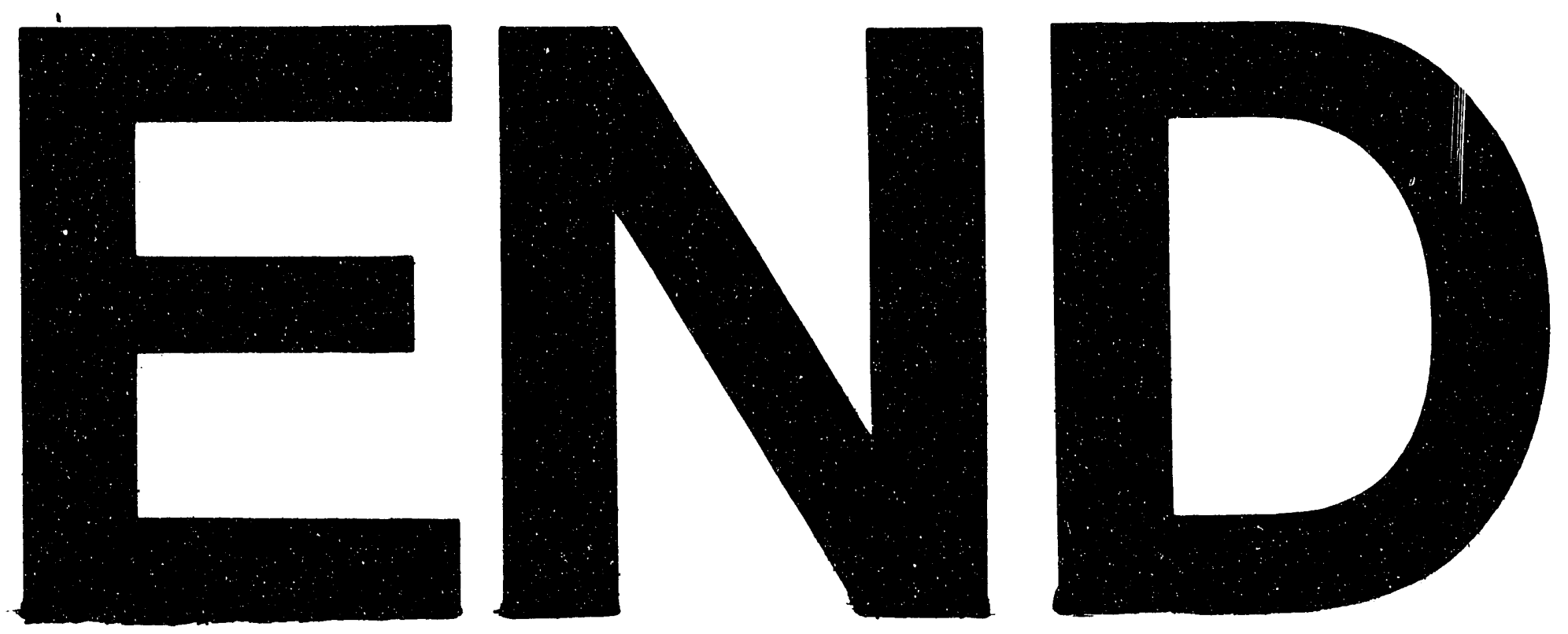

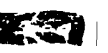

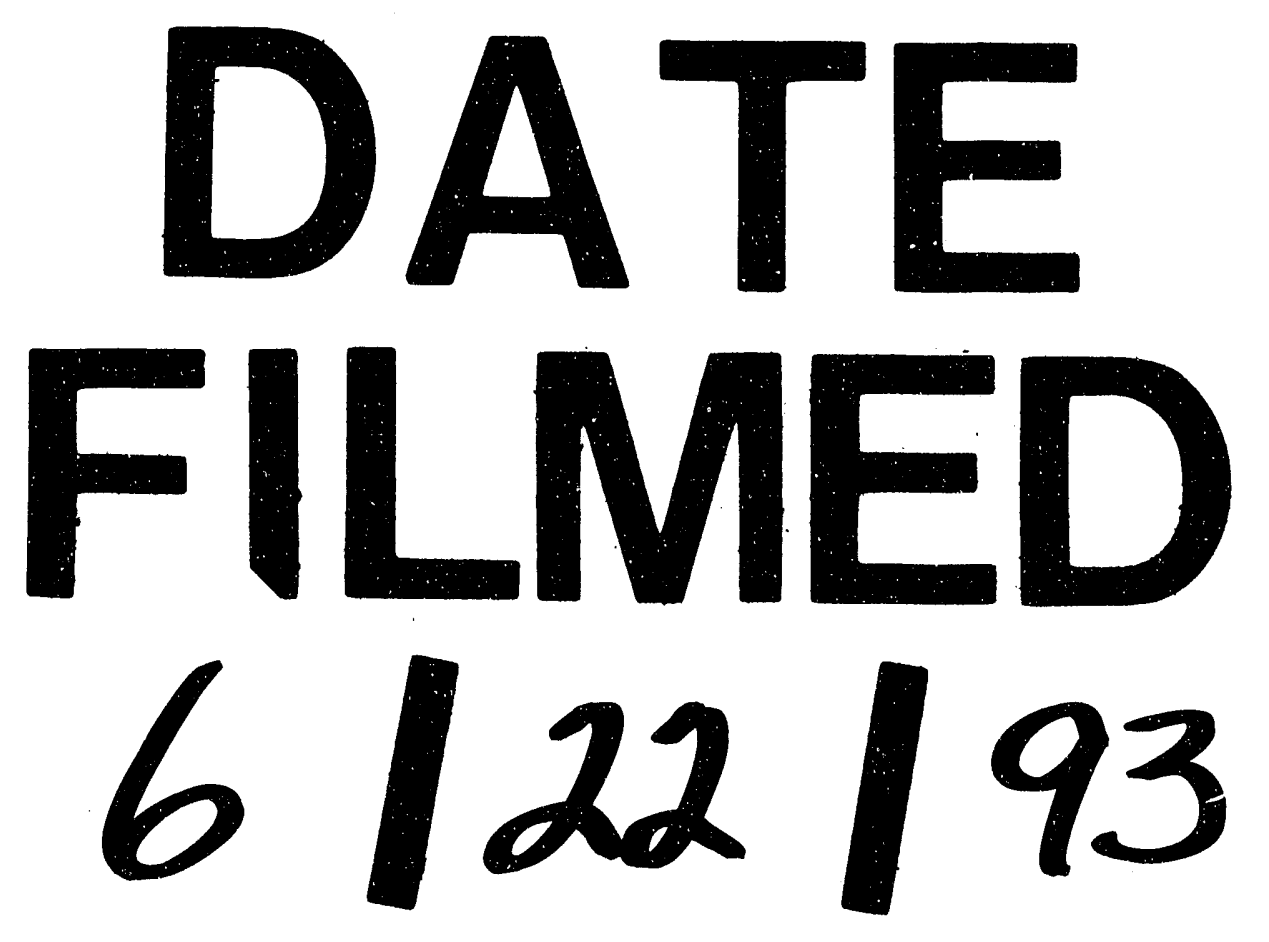


